\title{
Nano-Material Aspects of Shock Absorption in Bone Joints
}

\author{
H. Tributsch*,a, F. Copf ${ }^{\mathrm{b}}$, P. Copf $^{\mathrm{b}}$, U. Hindenlang ${ }^{\mathrm{c}}$, F.U. Niethard ${ }^{\mathrm{d}}$ and R. Schneider ${ }^{\mathrm{e}}$
}

${ }^{a}$ Retired from Free University Berlin, Institute for Physical and Theoretical Chemistry, 14195 Berlin, Takustrasse 3, Germany

${ }^{b}$ Bionic GmbH Schloss Monrepos, Gebäude 1, D-71634 Ludwigsburg, Germany

${ }^{c}$ Lasso Ingenieurgesellschaft GmbH, Leienfelderstr. 60, D-70771 Leienfelden-Echterdingen

${ }^{d}$ RWTH Universitätsklinik, Orthopädie, Pauwelstr. 3ß, D-52074 Aachen

${ }^{e}$ High Performance Computing Center, D-70569 Stuttgart, Nobelstr, 19

\begin{abstract}
This theoretical study is based on a nano-technological evaluation of the effect of pressure on the composite bone fine structure. It turned out, that the well known macroscopic mechano-elastic performance of bones in combination with muscles and tendons is just one functional aspect which is critically supported by additional micro- and nano- shock damping technology aimed at minimising local bone material damage within the joints and supporting spongy bone material. The identified mechanisms comprise essentially three phenomena localised within the three-dimensional spongy structure with channels and so called perforated flexible tensulae membranes of different dimensions intersecting and linking them. Kinetic energy of a mechanical shock may be dissipated within the solid-liquid composite bone structure into heat via the generation of quasi-chaotic hydromechanic micro-turbulence. It may generate electro-kinetic energy in terms of electric currents and potentials. And the resulting specific structural and surface electrochemical changes may induce the compressible intra-osseal liquid to build up pressure dependent free chemical energy. Innovative bone joint prostheses will have to consider and to be adapted to the nano-material aspects of shock absorption in the operated bones.
\end{abstract}

Keywords: Bone joint prostheses, spongy bone material, nano-material properties, pressure induced chemistry, shock absorption.

\section{INTRODUCTION}

Bone joint prosthesis such as total hip replacement has developed to become a medical success story with a routine activity within a multi-billion dollar business with approximately 300.000 hip replacements and even more knee replacements performed in the US annually alone at costs of up to $45000 \$$ each. A main focus of medical research has been to implant an artificial device of solid material components to provide mechanical stability and dynamic mobility while fixing it to the still stable rest of the bones. The artificial materials used to make the joints active again include ivory, metals such as stainless steel, tantalum or titanium, plastic material such as Teflon, polyethylene or acrylic bone cement, and ceramic material such as hydroxyl apatite ceramics, or combinations of them. While longer and longer life times for bone joint prosthesis could be achieved, various problems are known besides of dislocation of the hip or loosening of the prosthesis from the bone. There is the danger of infections around the implants and there is occasionally problematic wear debris, especially metal particulate debris, bone material degradation around the implant, necrotic bone tissue, and there are pseudo-tumours. Most important, the observation is made, that prosthetic joints are generally not as strong, reliable and durable as

*Address correspondence to this author at the San Leopoldo 47, 33016 Pontebba, Italy, Te1/Fax: 0039402890684; E-mail: helmut.tributsch@alice.it healthy natural joints. Patients are informed to avoid repetitive heavy lifting, excessive stair climbing and any high impact sports in order to increase the longevity and success of a hip replacement. They also should avoid activities involving quick stop-start motion, activities which were crucial for survival during evolution. The conclusion is that present bone joint prostheses do not match natural joints in durability and strength. The discovery and analysis of a sophisticated fine structure of the exposed membrane of the femur head and the isolation and study of the contained intraosseal fluid stimulated a re-evaluation of biological shock absorption and mechanical stress management. There must be physical-chemical mechanisms, that allow natural bone joints to be exposed to higher dynamic mechanical stress, that can not be solely matched with the mechanical performance of sophisticated artificial standard prosthesis.

A main task of this work will be to find out why present artificial bone joint prosthesis is inferior to natural bone joints both in performance and durability. Nano-composite material properties of the spongy bone material will be invoked and analysed, which are not jet sufficiently considered for the design of present-day artificial bone joint prosthesis.

\section{BONES - SEEN AS NANO-STRUCTURED DEVICES}

Evolution, during hundreds of million years, has extremely optimized the shock absorbing properties of the skeleton and of bone joints. The macroscopic 
accomplishments, the synergy of bone elasticity, muscles and tendons, in optimising movements, handling shock absorption and avoiding mechanical damage, has amply been investigated (e.g. [1, 2]) But what did nature accomplish with respect to static and dynamic bone stability on the microscopic scale within the bones and bone joints made up of highly structured solid-liquid composite nano-materials including hydroxyapatite, collagen, proteins and other organic substances? Mineralized collagen fibrils are made up of strings of alternating collagen molecules and equally sized hydroxyapatite crystals. These strings are stacked together in a staggered fashion giving rise to a hierarchical material structure. Weak and strong chemical bonds are alternating, and much room for liquid and organic material is left between solid particles. When pressure is applied, weak bonds break and liquid nano-spaces contract. It has been estimated that the internal surface of human bones alone, as provided by Harvesian canals, lacunae and canaliculi, may ten times exceed the internal surface of our lungs, which evolved for maximal exposure to oxygen (compare the three dimensional spongy bone structure demonstrated in Fig. 1). A pronounced three dimensional cavity generating structure is also apparent from the observation that an applied pressure of $2400 \mathrm{~N} / \mathrm{cm}^{2}$ or $24 \mathrm{MPa}$ is able to reduce the volume of the dried "substantia spongiosa" of the bone to $17 \%$ [3]. Nevertheless, the compression strength of living bone is greater than that of reinforced concrete (in excess of 50 $\mathrm{MPa}$ ) and its tensile strength approaches that of reinforced concrete. It appears that the fully functioning living bone has mechanical properties clearly exceeding those of dried bone material. But established prosthesis technology was not aiming at matching the function of living bone, because this aspect remained largely unexplored.

These nano-material aspects of bones, which correlate with exceptionally large surface areas and a composite solidliquid structure, cannot be accidental and became especially evident when repairing them with traditional hip or femur prostheses. It turned out that the interaction of the artificial material, which is non permeable against liquid, with the living bone structure is problematic, sometimes causing bone degradation within several years. The micro- and nanostructure as well as the dynamics of the spongy solid-wet bone material does not match well with artificial solid metallic, ceramic and plastic implants. Instead of healing and growing, the bone material directly exposed to the prosthesis may degrade. If the performance and the survival time for implants could be significantly extended, medical expenses of billions of dollars could annually be avoided and patients could better adapt to their situation.

Over the years more attention has been paid to the differences between micro and nano- porous bone joint interfaces and available technical metallic, plastic or ceramic prosthetic interfaces. The aim was to learn from the natural example of a nano-material dominated function in bones (Fig. 1) by following a bionic strategy towards improved artificial joints. They should be designed to tolerate and stimulate natural nano-technological bone function. Important discoveries were a specialized microstructure within the involved porous bone material on the femur surface [3] and its penetration by an intraosseal liquid [4, 5]. But microstructure continues into the spongy bone material. It is made up of collagen-hydroxyapatite mineral matrix, which is infused with free spaces, lacunae, and canals. Its structure and function is, as well known, controlled by three types of biological cells dominating bone homeostasis: the bone building osteoblasts, the stationary osteocytes, and the bone destroying osteoclasts. Osteocytes are ideally positioned within the bone to sense mechanical strain and to translate it into biochemical signals that control bone transformation such as the deposition and removal of minerals from lacunae and canaliculi. They are also responsible for phosphate homeostasis which suggests the control of multiple regulatory mechanisms [6]. The challenge is to understand the function of this highly structured biology controlled solid-wet composite mechanosensitive bone tissue (compare Fig. 1), which is known to adapt functionally to varying levels of mechanical stress, in terms of nano-material technology. Fig. (1) is intended to visualize the complex three dimensional composite structure of bones. With the inserted SEM pictures it gives an impression of the high degree of microscopic structuring of a bone. The pictures show structures in the micrometer range, but structuring continues down to the nano-scale where hydroxyl-apatite crystals and collagen fibres are forming a well organized composite material.

The large internal surfaces of the micro-and nanostructured bone matrix as well as the included liquid filled nano spaces allow diverse new interpretations of pressure induced mechanisms:

\section{NANO-MECHANISMS FOR MECHANICAL ENERGY DISSIPATION}

As Fig. (1) shows, bones have a very elaborate microscopic fine structure. What kind of shock absorbing mechanisms could have developed within? The tensulae, for example [3,5] which were identified within the pressure exposed outer surface of the femur head, are apparently elastic membranes which compartmentalize the porous bone structure further and allow the enclosed liquid to escape through small openings, when pressure is applied. Such a nano and micro-structural internal bone geometry and behaviour may be characterized schematically and in a simplified way by small cylinders, equipped with little openings, as used in macroscopic hydraulic shock absorbers, where a piston is decreasing the liquid volume and forcing it through the openings while dissipating mechanical energy into heat. Such energy dissipating small liquid filled cylinders are schematically shown in Fig. (2) to represent in a simplified way the bone structure during a mechanical shock generating the temporal pressure difference of $\Delta \mathrm{p}$. When the spongy bone structure containing the liquid filled compartment is compressed, the intra-oseal liquid will redistribute through the escape openings and thus dissipate mechanical energy while liberating heat. This is schematically indicated at the bottom right of Fig. (2). Due to the microscopic structure of the described energy dissipating system the conversion of a stationary liquid into a quasi chaotic turbulent one is to be expected.

Pressure dependent chemical reactions are also expected to occur within the intra-oseal liquid. Such reactions are only significant when products split up into positive and negative charges such as $\mathrm{HA} \rightarrow \mathrm{H}^{+}+\mathrm{A}^{-}$. Phosphate compounds, which in the bone are present as hydroxylapatite 


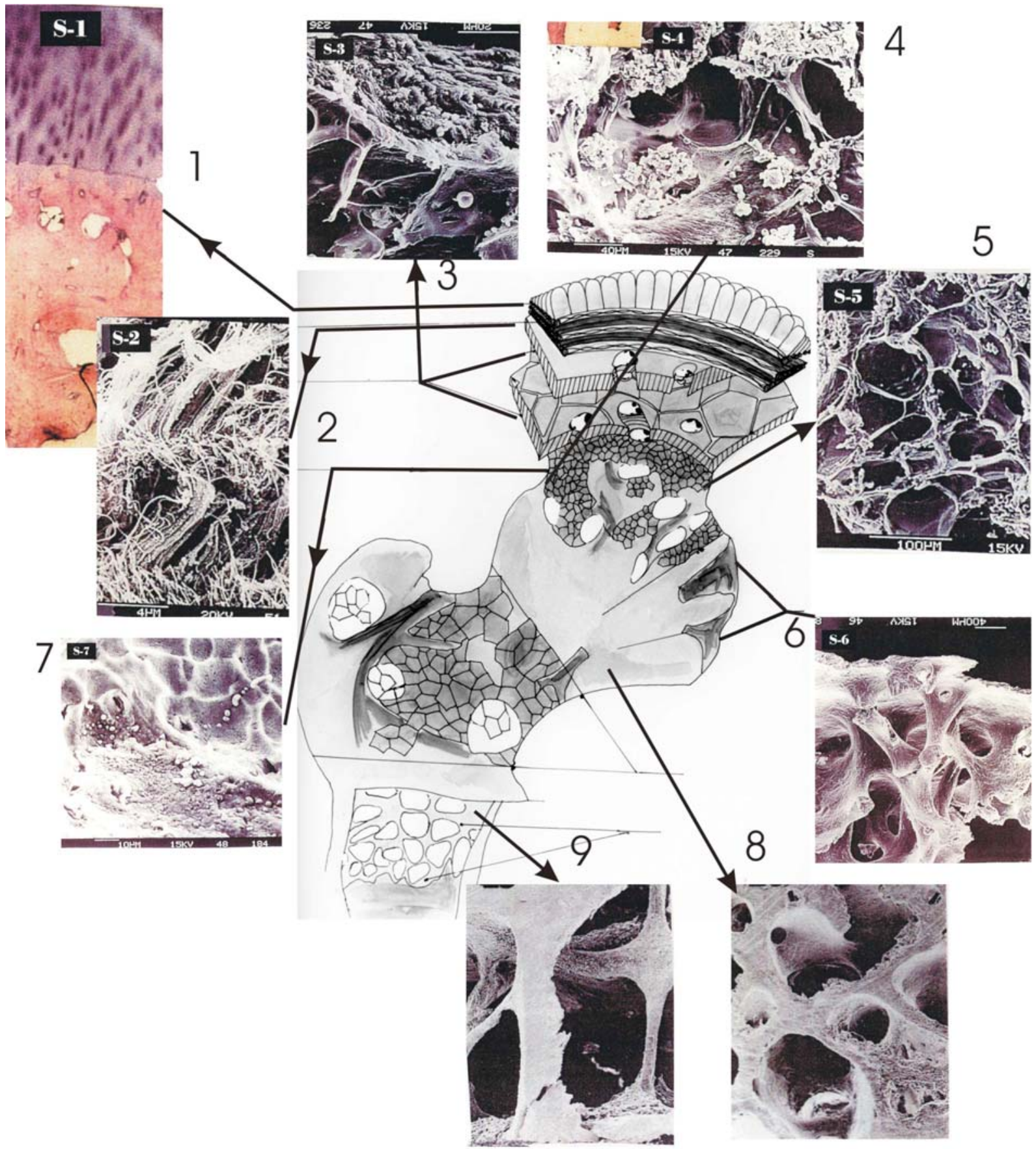

Fig. (1). shows a schematic drawing of a bone with its most characteristic fine structure (shown as SEM pictures with micrometer resolution): 1: cartilage, 2: tide -marrow, 3: calcified zone, 4: subchondriala compacta, surface of femur head, 5: cysterns between calcified zone, 6: subchondriala compacta (inverted), 7: epi-line (epiphysal joint): 8: Paul`s pressure-tension trajectories, 9: transition between joint and diaphysis.

$\left(\mathrm{Ca}_{10}\left(\mathrm{PO}_{4}\right)_{6} \mathrm{OH}_{2}\right)$ and also make up a significant portion of intra-oseal liquid, belong to this group. They may dissociate like $\mathrm{H}_{3} \mathrm{PO}_{4}=\mathrm{H}^{+}+\mathrm{H}_{2} \mathrm{PO}_{4}^{-}$or $\mathrm{Ca}_{3}\left(\mathrm{PO}_{4}\right)_{2}=3 \mathrm{Ca}^{2+}+2 \mathrm{PO}_{4}{ }^{3-}$. In this case the solvate layer around the liberated ions is reorganized and water molecules are more densely packed resulting in a volume decrease. The consequence is a pressure-induced free energy storage in the system. The chemical properties of the intra-oseal liquid have been studied in some detail ${ }^{7}$. Major phosphorus $\left({ }^{31} \mathrm{P}\right)$ NMR signals were indeed found. Compressibility of the liquid was obtained under alkaline conditions. The neighbourhood of nano-crystalline hydroxyapatite and phosphate containing liquid in the spongy bone substance can not be accidental. Hydoxyapatite is now widely used as a highly selective substrate for chromatography since it provides very effective $\mathrm{PO}_{4}{ }^{2-}$ and $\mathrm{Ca}^{+}$adsorption sites [8]. It is also remarkable that phosphate buffers are widely used to produce a liquid environment for generating combined pressure and $\mathrm{pH}$ jumps in scientific studies. Such a pressure dependent phosphate containing solution also characterizes the 
environment of hydroxyapatite-collagen nano-structures of bones. Evolution has indeed selected a pressure-active environment for optimising bone function. It can only be intended for shock absorption within the bone structures! This environment can absorb mechanical energy by converting it into chemical energy and it may also serve for additional energy-relevant purposes.

The microscopic structure of spongeous bone material suggests additional pressure related mechanisms. It is, for example, known that pressure changes in bones induce piezoelectric and dielectric phenomena which are essentially attributed to the collagen fraction of the collagenhydroxyapatite nanostructure [9]. It may be speculated that pressure induced electrochemical changes in the bone structure can induce an appropriate $\mathrm{pH}$ change to get a pressure induced temporary increase of stored free chemical energy in the liquid and around the hydroxyapatite particles. This chemical energy may subsequently either be released as heat or recaptured as chemical energy by still unknown following reactions.

Referring the pressure $\mathrm{p}$ attained in the joint (up to 50$100 \mathrm{MPa}$ ) to the atmospheric pressure, the expected free energy $\mathrm{G}$ of the intra-oseal liquid is ( $G_{0}=$ free energy at one bar)

$G=G_{0}+R T \ln p$

The resulting change of the equilibrium constant $\mathrm{K}$ can then be calculated to

$\frac{\Delta K}{K}=-\frac{\Delta P \Delta V}{R T}$

where $\triangle P \Delta V$ is the mechanical energy involved in the shock. A pressure dependent shift in the equilibrium constant, with a corresponding increase of chemical products, with possible precipitation reactions, as well as a pressure dependent free energy change are consequently to be expected. This behaviour is schematically indicated in Fig. (2) at the bottom centre. A comparable behaviour as discussed for bone liquid was observed with model milk salt solutions where pressure generates significant shifts in their colloidal and soluble $\mathrm{Ca}$ phosphate equilibrium and concentration, $\mathrm{pH}$ and casein micelle microstructure [10]. A similar pressure jump phenomenon is to be expected in the solution-permeated bone nanostructure, where interfacial phosphate will dissociate due to a shift of the equilibrium constant (relation (2)). It is obvious, that the increase of bone density caused by increased sustained pressure application to bones may equally be related to pressure induced shifts of chemical equilibria (relation (2)).

A third phenomenon expected in bones and bone joints are piezoelectric and electrokinetic effects (Fig. 2, bottom). The first arise from the collagen in the bone tissue and the second from pressure induced changes in the double layer surfaces of the wet composite material. Electrical currents as well as potentials are generated which in turn may induce changes in the ion concentration and, due to solvate reorganization, of the liquid's volume. The ultimate consequence will be the conversion of mechanical energy into heat with electrical and chemical energy as intermediate forms of stored energy.

All three discussed pressure dependent reactions may superpose and interfere. They take away energy peaks from the mechanically stressed system, thus decreasing the exposure to material deteriorating processes.

With respect to the liquid associated with porous bone structures there is an additional factor which also has to be considered:

If a transient positive pressure maximum is passing through a liquid-soaked porous material, it may be followed by a minimum of pressure, a situation of negative pressure (tension). The consequence of tension in the liquid would be cavitation, with a temporary formation of water vapour bubbles and their subsequent destructive collapse. Such cavitation events are known to destroy even the metal of ship screws, if they are not properly designed, and would also destroy bone material, if they would occur within bones due to mechanical shock events. Since functioning bones are very long lived, cavitation in the bone composite material is apparently suppressed. Such a suppression of cavitation is known to occur in other biological systems, for example in the sap transporting conduits of trees. Liquids in appropriately optimised microscopic tree capillaries are able to build up and sustain negative pressures or tensions. This is well known from the water transport system in the Xylem of trees where tensions of several Megapascale (MPa) may build up and be sustained. This is the reason why trees can extract water from arid soil, and pump it over 100 meters high as known to occur in Eucalyptus and Sequoia trees. A liquid in micro-conduits that can sustain tensions without cavitation is also able to oscillate [11] and could thus significantly improve the elastic behaviour and stability of a shock absorbing semi-wet composite material. Much research will be needed to understand these complicated pressure and tension related mechanisms in nano-and microstructured bone material. The presently available computational tool of sophisticated finite element analysis of strain in bones [12] could probably be used to gain experience with nano-material bone properties. Correspondingly adapted pressure and time dependent elastic and energetic parameters would have to be considered in the model calculations.

\section{CHALLENGES FOR PROSTHESIS RESEARCH}

Traditional bone joint prosthesis research did essentially aim at reproducing macroscopic mechanic performance by integrating the prosthesis into the apparently most stable still functioning bone structures. Properties and functions of spongeous bone material were essentially ignored. Most of the spongeous material was cut away as it was considered mechanically unstable. But as this study shows, its complex nano-material behaviour could be essential for approaching biological bone joint quality. It actively contributes to the energetic and mechanical properties of bone function. A straightforward consequence of this nano-material interpretation of mechanical bone properties would be that, whenever possible, artificial replacements of bone joints should aim at conserving, developing and amplifying the nano-technological mechanisms involved. 


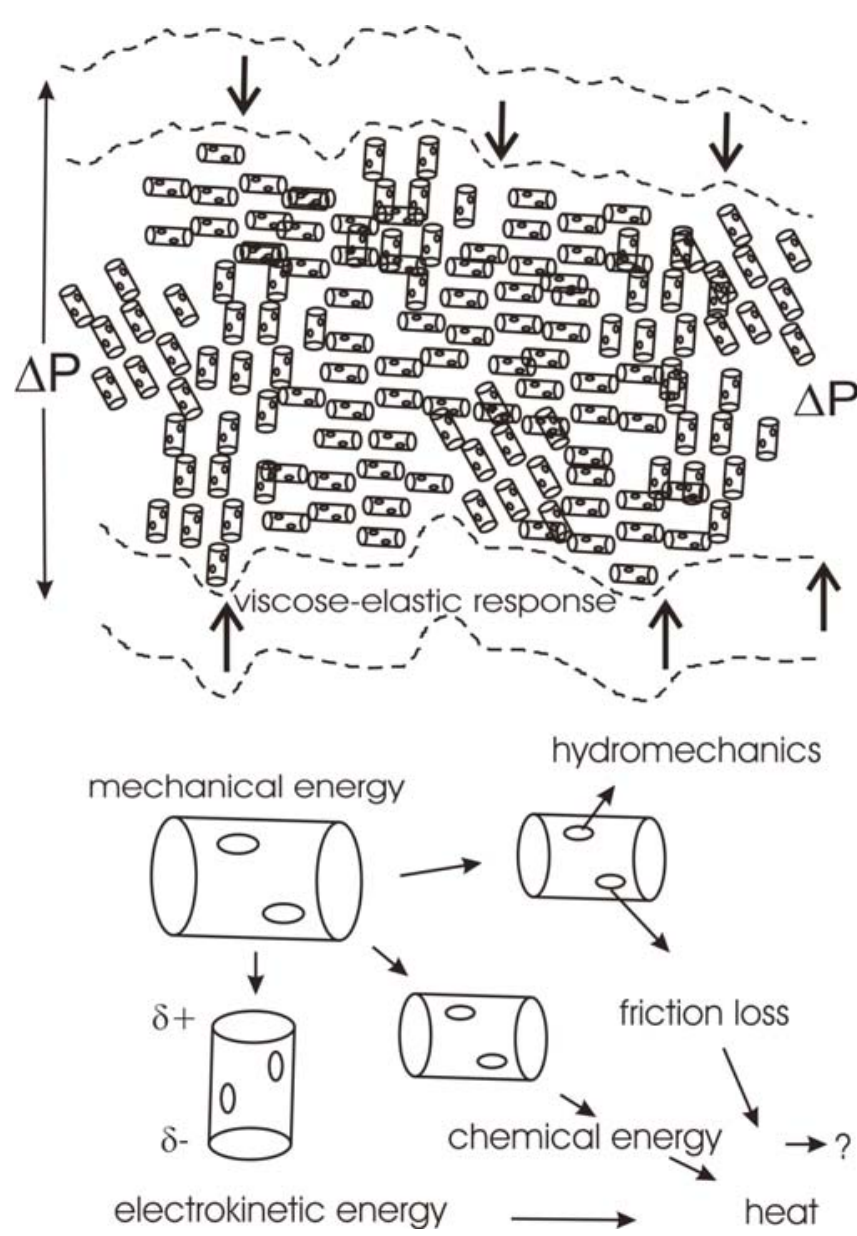

Fig. (2). Scheme, visualizing three mechanisms for the dissipation and transformation of mechanical energy in solid-liquid nanocomposite layers of bone joints subject to a pressure difference of $\Delta \mathrm{P}$.

Modern hip prosthesis will have to be adjusted to take advantage of nature's nano-technological accomplishments towards shock absorption. Spongiosa-supported shock absorption should be a key aim of advanced applied research. Material and form of the prosthesis must be designed in such a way as to support and to promote nanoactivity. It should avoid destruction of natural nanocomposite, spongeous bone material in its vicinity and allow recovery and build-up of actively functioning bone substance.

Essential steps in this direction have been initiated two decades ago with modern prototypes of bio-analogue prosthesis for humans [13-16] based on preceding experiments with dogs, which demonstrated that the spongiosa material of bones can actually be incorporated and used to support shock absorption in the environment of the prosthesis ([17] and application of this concept to the design of innovative prosthesis [18]). However today, with traditional artificial joints, the bond between the bone and the prosthetic material still frequently breaks down and fatigues and fragments of wear debris may cause inflammatory reaction. The spongy bone material with its specific nano-material function obviously does not properly match with the solid steel, ceramic or plastic material of the implants [19]. The consequence is pain and a reduction of movements with the result of a weakening of muscles [20]. A characteristic problem is also osteolysis, presently understood as autoimmune reaction resulting from the body's attempt to clean up the wear particles formed within the bone tissues [21]. A similar problem of osteolysis is known to arise in the highly stressed shoulders of weight lifters [22]. This shows that presently used moderately stressed artificial joints apply a too high, not effectively attenuated pressure to the bone material. It reacts as if an excessively high pressure, as in the shoulders of weight lifters, would prevail. Many publications in the field of joint surgery reflect additional complications which are presently not well understood [23]. This supports our considerations of a nano-material function of spongy bone material, which is not tolerated and supported by typical present-day implants. A principal aim for construction and implantation of a joint prosthesis should be to maximally conserve the volume of spongeous bone material by correspondingly optimising the shape (minimal volume and perforated and communicating form of implant) of the implanted prosthesis and by adjusting its interfacial properties in order to support nanobiological activity. These interfacial properties include adapted physical-chemical properties and a maximised (ideally fractal or net-shaped) contact surface to minimise the transmitted pressure.

In order to achieve the goal of a high standard prosthesis based on bio-analogue nano-bone technology significantly more research and testing will be needed to better understand the biological mechanisms involved and to identify relevant artificial nano-composite material structures for bio-analogue prostheses. For this purpose advantage of the strategy should be taken to incorporate sensors into artificial bone joints to monitor relevant parameters during biological mechanical activity. This way we may gradually learn why artificial prostheses, in spite of the use of high quality technical material, can not yet match natural bone joint technology.

\section{REFERENCES}

[1] R. McNeill Alexander, The Unity of Form and Function, Mc Millan, 1994.

[2] S. Vogel, Comparative Biomechanics: Life's Physical World, Princeton University Press, 2003.

[3] F. Copf, A. Czarnetzky, and W.Lierse, "Substrukturen in der Substantia spongiosa des Caput femoris aund des Talus", Acta Anat. vol. 138, pp. 297-301, 1990.

[4] J.A. Ochoa, D.A. Heck, B.M. Hillberry, and K.E. Brandt, "The effect of osseous fluid on the mechanical behaviour of femoral heads", Transactions of the $34^{\text {th }}$ Meeting of the Orthopaed. Res. Soc., Vol. 13, pp. 126, Feb 1988.

[5] E. Petresin, "Membransystem der Tensulae, intraosseale nonNewton Flüssigkeit als Teil des hydrodynamischen Dämpfers im spongiösem Knochen", Proceedings of Bionic-Workshop, Bistra, Slovenia 30/31.05.2003, ISBN 961-6464-06-X (Techniski musej Slovenije), May 2003.

[6] J. Bonewald, "Osteocytes as multifunctional cells", $J$. Musculoscelet. Neuronal Interact., vol. 6, pp. 331-333, 2006.

[7] J. Plavee "Characterisation of Prof. Ravnik's Samples by NMR Spectroscopy"; Bionic-Workshop, Bistra, Slovenia 30/31.05.2003, ISBN 961-6464-06-X (Techniski musej Slovenije), May 2003.

[8] P. Gagnon, "An enigma unmasked: How hydoxylapatite works and how to make it work for you". Validated Biosystems Quarterly Resource Guide for Downstream Processing, retrieved from: http://www.validated.com on 12/6/2010.

[9] H. Maeda, K. Tsuda, and E. Fukada, "The dependence on temperature and hydration of piezoelectric and elastic constants of bone", Jpn. J. Appl. Phys., vol. 15, pp. 2333-2336, 1976. 
[10] K. Schrader, W. Buchheim, and CV Morr, "High pressure effects on the colloidal calcium phosphate and the structural integrity of micellar casein in milk", Nahrung, vol. 4, pp. 133-138, 1997.

[11] H. Tributsch, J. Cermak, and N. Nadezhdina. "Kinetic Studies on the Tensile State of Water in Trees", J. Phys. Chem. B, vol. 109, pp. 17693-17707, 2005.

[12] R. Schneider, U. Hindenlang, and P.Copf, "Dynamic finite element analysis of cancerous bone microstructure", in press 2010.

[13] F. Copf, U. Holz, and. S. Vesel, "Eine biomechanische Lösung zur dauerhaften Verankerung künstlicher Hüftgelenkpfannen" Zeitschrift für Orthopädie, vol. 121, pp. 265-270, 1983.

[14] R.Coombs, A. Gristina, and D. Hungerford, Joint Replacement, State of the Art, Introduction, XXXIII, Fig. 3, Orthonext, London, 1990.

[15] F. Copf, and U. Holz, Knochen als dynamisches Prinzip, Georg Thieme Verlag Stuttgart, 1994.

[16] F. Copf, Workshop zur Krafteinleitung und bionische Endprothese, Bionic- workshop, Bistra, Slovenia, 30./31. 5. 2003, ISBN 9616464-06-X (Techniski musej Slovenije), May 2003.

[17] F. Copf, and A Czarnetzki, "Detection of two membrane systems in the subhondral zone of the head of femur", MED RAZGL (Slovenia): vol. 28, pp. 95-106, 1989; and lecture given at Ljubljana University, considering bone hydrodynamic data, and spongiosa supported shock absorption, obtained in experiments (with necrotic bones of dogs) in collaboration with F.U. Niethard.

[18] U.Holz, F. Copf, G. Faust, and W. Lierse, "Hüftprothesenimplantation unter Berücksichtigung neuer Strukturen im Skelettsystem“, Hefte zu „Der Unfallchirurg“, Heft 261, E. Schneider (editor), Biomechanik des menschlichen Bewegungsapparates, Springer, Berlin, Heidelberg, 1997.

[19] Joint Replacement Surgery and You. (April, 2009) In Arthritis, Musculoskeletal and Skin Disease online. Retrieved from http://www.niams.nih.gov/\# on 30/7/2010.

[20] Your Orthopaedic Connection: Total joint replacement (July, 2007) In American Academy of Orthopaedic Surgeons online. Retrieved from http://www.aaos.org/ on 30/07/2010.

[21] S. Agarwal, "Osteolysis - basic science, incidence and diagnosis". Current Orthopaedics, vol. 18, pp. 220-231, 2004, doi:10.1016/j.cuor.2004.03.002.

[22] R. Schwarzkopf, C. Ishak, M. Elman, J. Gelber, N.D.Strauss, and L.M. Jazrawi, "Distal clavicular ostolasis: a review of the literature", Bull. NYU Hosp. Jt. Dis., vol. 66, no. 2, pp. 94-101, 2008.

[23] M. Hamadouche, P. Boutin, J. Daussange, M.E. Bolander, and L. Sedel, "Alumina-on-alumina total hip anthroplasty", J. Bone Joint Surg., vol. 84, pp. 69, 2002.

(C) Tributsch et al.; Licensee Bentham Open.

This is an open access article licensed under the terms of the Creative Commons Attribution Non-Commercial License (http://creativecommons.org/licenses/by-nc/3.0/) which permits unrestricted, non-commercial use, distribution and reproduction in any medium, provided the work is properly cited. 